HU-EP-08/25

\title{
Matching gluon scattering amplitudes and Wilson loops in off-shell regularization
}

\author{
Harald Dorn, Charlotte Grosse Wiesmann \\ Humboldt-Universität zu Berlin, Institut für Physik \\ Newtonstr. 15, D-12489 Berlin \\ dorn@physik.hu-berlin.de, lottagw@yahoo.de
}

\begin{abstract}
We construct a regularization for light-like polygonal Wilson loops in $\mathcal{N}=4$ SYM, which matches them to the off-shell MHV gluon scattering amplitudes. Explicit calculations are performed for the 1-loop four gluon case. The off light cone extrapolation has to be based on the local supersymmetric Wilson loop. The observed matching concerns Feynman gauge. Furthermore, the leading infrared divergent term is shown to be gauge parameter independent on 1-loop level.
\end{abstract}




\section{Introduction}

The mapping of planar gluon MHV scattering amplitudes in $\mathcal{N}=4$ SYM to Wilson loops for polygons built out of light-like segments and their evaluation via related string configurations at strong 't Hooft coupling [1] has widened the toolkit of AdS/CFT considerably. Soon after this, it has been realized that the correspondence between scattering amplitudes and Wilson loops also holds at weak 't Hooft coupling as a property of perturbative gauge field theory [2-4]. Both partners of the correspondence are divergent. Therefore, one has to specify regularizations on both sides to get a precise statement out of the AdS/CFT lore, which is behind this correspondence. So far this has been done with dimensional regularization on both sides, matching its dual IR/UV aspects.

Given the need to specify a regularization, it is a natural question to ask how robust the correspondence is with respect to the choice of regularizations. For scattering amplitudes, besides dimensional regularization, there is another very natural regularization: going off shell with the external momenta. In ref. [2] it has been stressed, that this off-shell regularization is sensible to a larger soft kinematical region than dimensional regularization, resulting in a characteristic factor 2 in the evolution equation. This observation gives an additional motivation to find the corresponding regularization on the Wilson loop side.

The aim of this short note is to identify exactly this wanted regularization out of the study of the simplest case, the four-gluon amplitude in one loop approximation. Since the construction will turn out to be very natural, we conjecture its validity in general.

The quantity which now has to be matched by the Wilson loop calculation is the one-loop four-gluon amplitude (divided by the tree amplitude) with all four momenta slightly off shell, i.e. $p_{i}^{2}=-m^{2},[2]$

$$
\mathcal{M}_{4}=1+a M^{(1)}+\mathcal{O}\left(a^{2}\right)
$$

with

$$
a=\frac{g^{2} N}{8 \pi^{2}}
$$

and ( $s, t$ Mandelstam variables)

$$
M^{(1)}=-\log ^{2}\left(\frac{m^{2}}{-s}\right)-\log ^{2}\left(\frac{m^{2}}{-t}\right)+\frac{1}{2} \log ^{2} \frac{s}{t}-\frac{\pi^{2}}{6}+\mathcal{O}(m) .
$$

\section{The Wilson loop partner for off-shell scattering amplitudes}

A first option could be to keep the identification of the sides of the tetragon with the momenta of the scattering amplitude also taken off shell. While this makes the scattering amplitude finite, the corresponding Wilson loop is still divergent due to the cusp divergences (now cusps between space-like sides). If we handle these by dimensional regularization $(D=4-2 \epsilon)$ and treat the $\mathrm{RG}$ scale parameter as in [2], 
i.e. $g^{2} \rightarrow g^{2}\left(\mu^{2} \pi e^{\gamma}\right)^{-\epsilon}$, we get

$$
\mathcal{W}_{4}=1+a W^{(1)}+\mathcal{O}\left(a^{2}\right)
$$

with

$$
\begin{aligned}
\left.W^{(1)}\right|_{\text {off-cone, } \epsilon=} & \frac{1}{\epsilon}\left(\log \left(\frac{\mu^{2}}{-s}\right)+\log \left(\frac{\mu^{2}}{-t}\right)+2 \log \left(\frac{m^{2}}{\mu^{2}}\right)\right)+\log ^{2}\left(\frac{m^{2}}{\mu^{2}}\right)-\frac{1}{2} \log ^{2}\left(\frac{\mu^{2}}{-s}\right) \\
& -\frac{1}{2} \log ^{2}\left(\frac{\mu^{2}}{-t}\right)+\frac{1}{2} \log ^{2}\left(\frac{s}{t}\right)+\frac{\pi^{2}}{2}+\mathcal{O}\left(m^{2} \log m\right)+\mathcal{O}(\epsilon) .
\end{aligned}
$$

Suppressing the pole term in $\epsilon$ yields the renormalized $\left.W^{(1)}\right|_{\text {off-cone, ren, which depends }}$ on $\mu^{2}$ and $m^{2}$. For our purpose it seems natural to identify $\mu^{2}$ and $m^{2}$, which results in 1

$$
\left.W^{(1)}\right|_{\text {off-cone, ren }}=-\frac{1}{2} \log ^{2}\left(\frac{m^{2}}{-s}\right)-\frac{1}{2} \log ^{2}\left(\frac{m^{2}}{-t}\right)+\frac{1}{2} \log ^{2}\left(\frac{s}{t}\right)+\frac{\pi^{2}}{2}+\mathcal{O}\left(m^{2} \log m\right) .
$$

Obviously, this does not match (3), the main obstacle is the factor $\frac{1}{2}$ in front of the terms quadratic in $\log m^{2}$. This remains true also if one keeps $\mu^{2}$ fixed and looks at $m^{2} \rightarrow 0$. As a side remark, note that the difference in the finite terms independent of the Mandelstam variables, could even be removed by including a suitable numerical factor in the relation between $\mu^{2}$ and $m^{2}$.

The mismatch cannot be avoided either, by skipping the intermediate step via the renormalized Wilson loop. Trying this, one immediately would have to use in (5) the familiar mapping of dimensional regularization to regularizations with dimensional parameter: $\frac{1}{\epsilon} \sim-\log \left(\frac{m^{2}}{\mu^{2}}\right)$.

After this unsuccessful attempt, we stay in four dimensions and consider a combination of $p_{i}^{2}=-m^{2}$ with a suitable cutoff for the contour parameter $\tau$. As long as the sides of the polygon are light-like the scalars decouple. Therefore, one can use either the Wilson loop for gauge fields $A_{\mu}$ only, or the local supersymmetric Wilson loop coupling to $A_{\mu}$ and the scalars $\phi_{I}[5,6]$

$$
\mathcal{W}=\left\langle\frac{1}{N} \operatorname{tr} \mathrm{P} \exp \left(i \int\left(A_{\mu} \dot{x}^{\mu}+\sqrt{\dot{x}^{2}} \theta^{I} \phi_{I}\right) d \tau\right)\right\rangle .
$$

However, as soon as the sides are no longer light-like, one has to make a decision. 2 We choose the local supersymmetric loop (7), since then the divergences arising from the limit of coincident propagator end points in the interior of a side of the polygon cancel each other. One only has to specify a cutoff for the contour parameter near the cusps.

Cutting out part of the closed contour breaks gauge invariance. But this is no obstruction, since the partner on the side of the scattering amplitude is not gauge

\footnotetext{
${ }^{1}$ That this renormalized expression diverges for $m^{2} \rightarrow 0$ is a reflection of the divergence of the cusp anomalous dimension for space-like sides if the sides approach a light-like limit.

${ }^{2}$ Out of the formal construction according to AdS/CFT in [1], the local supersymmetric version seems to be the natural partner. Further support arises from the treatment of divergences.
} 
invariant off shell either. What we now try to match are quantities obtained by using Feynman gauge on both sides. We will add some more comments on gauge variance versus invariance below.

We normalize the dimensionless contour parameter $\tau$ by assigning an interval of length 1 to each side of the polygon. To relate the cutoff for the dimensionless $\tau$ to the dimensionful $m^{2}$, we need another dimensionful quantity to form a quotient. For the cusp spanned by the momenta $p_{j}$ and $p_{k}$ there is only the scalar product $p_{j} p_{k}$ available. Since $\tau$ characterizes a portion of momentum, the wanted quotient should be proportional to $m$ instead of $m^{2}$. Our choice for the cutoff in $\tau$ at the cusp spanned by $p_{j}$ and $p_{k}$ then is

$$
\sigma_{j k}=\sqrt{\frac{m^{2}}{-2 p_{j} p_{k}}} .
$$

The minus sign under the square root means that we first define our regularized Wilson loop in the kinematical region, where all scalar products $p_{j} p_{k}$ are negative 3 and then continue from this region analytically. For the four gluon scattering this region is just the $u$-channel.

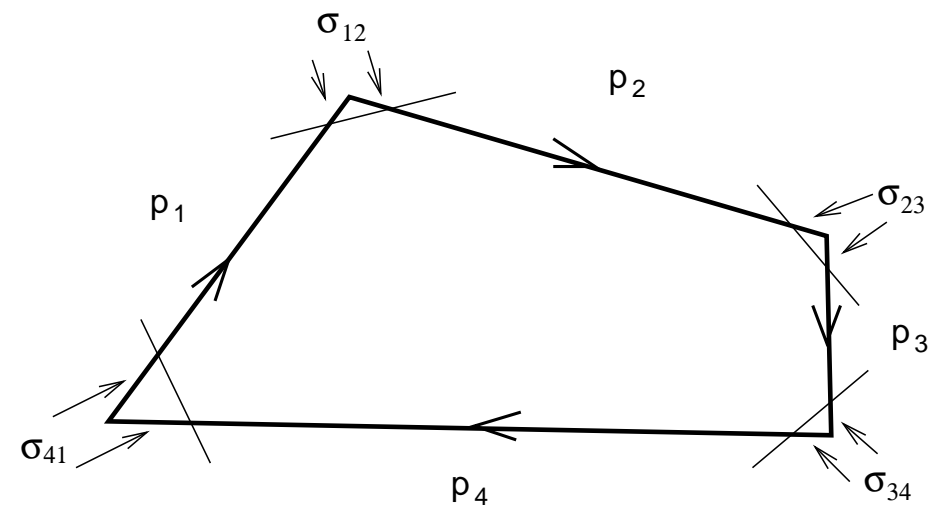

Fig.1 A tetragon contour for the Wilson loop with cut cusps.

There is still another motivation for the choice (8). For points $x_{j}, x_{k}$ on the $p_{j}$-side or $p_{k}$-side, respectively, let us first cut out the region

$$
\left|\left(x_{j}-x_{k}\right)^{2}\right| \leq m^{2} .
$$

Relating the forbidden Minkowski space distances directly to the infrared regulator of the scattering amplitude, this is a very natural starting prescription. With the parametrization $x_{j}=-\tau_{j} p_{j}, \quad x_{k}=\tau_{k} p_{k}$ and $\tau_{j}, \tau_{k} \in[0,1]$, in the $\left(\tau_{j}, \tau_{k}\right)$-plane, this corresponds to cutting the region (note: $p_{j}^{2}=p_{k}^{2}=-m^{2}, p_{j} p_{k}<0$ )

$$
\tau_{j}^{2}+\tau_{k}^{2}+\frac{\left(-2 p_{j} p_{k}\right)}{m^{2}} \tau_{j} \tau_{k} \leq 1
$$

out of the unit square. The crucial point is that here the quotient $\frac{\left(-2 p_{j} p_{k}\right)}{m^{2}}$ appears of its own volition. The hyperbola saturating the inequality in (10) has its nearest

\footnotetext{
${ }^{3}$ We have chosen the mostly minus metric.
} 
point relative to the origin at $\tau_{j}=\tau_{k}=\left(2+\frac{\left(-2 p_{j} p_{k}\right)}{m^{2}}\right)^{-1 / 2}=\sigma_{j k}\left(1+\mathcal{O}\left(\sigma_{j k}^{2}\right)\right)$. Since divergences arise only when both, $x_{j}$ and $x_{k}$, approach the cusp, eq.(9) cuts out too much. If we then use the behaviour of the hyperbolas in the vicinity of the origin to determine the size of the cut squares, we just get (8) for their length. In figure 2 we show the relation of the cut regions according to eqs.(9) and (10) in comparison to the above argued cut $\tau_{j}, \tau_{k} \leq \sigma_{j k}$ for various values of $\sigma_{j k}$.

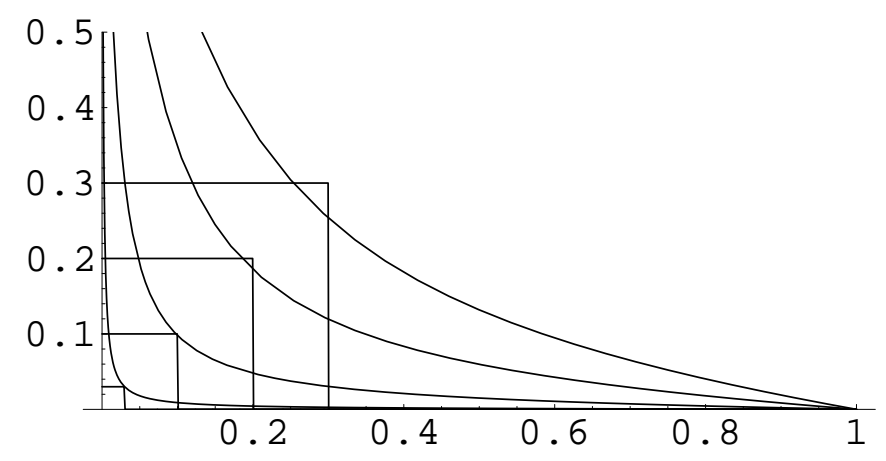

Fig.2 Cut regions in $\left(\tau_{j}, \tau_{k}\right)$-plane according to eqs.(9) and (10) compared to $\tau_{j}, \tau_{k} \leq$ $\sigma_{j k}$, for $\sigma_{j k}=0.3,0.2,0.1,0.03$, respectively.

After these preparations the one loop contribution to the local supersymmetric Wilson loop for the tetragon of Fig.1 is given by

$$
W^{(1)}=I_{12}^{\text {cusp }}+I_{23}^{\text {cusp }}+I_{34}^{\text {cusp }}+I_{41}^{\text {cusp }}+I_{24}+I_{13}
$$

with

$$
I_{j k}^{\text {cusp }}=\int_{\sigma_{j k}}^{1} d \tau_{j} \int_{\sigma_{j k}}^{1} d \tau_{k} \frac{p_{j} p_{k}+m^{2}}{-\left(\tau_{j} p_{j}+\tau_{k} p_{k}\right)^{2}+i \varepsilon}
$$

and

$$
I_{24}=\int_{0}^{1} d \tau_{2} \int_{0}^{1} d \tau_{4} \frac{p_{2} p_{4}+m^{2}}{-\left(\tau_{2} p_{2}-\left(p_{2}+p_{3}+\tau_{4} p_{4}\right)\right)^{2}+i \varepsilon} .
$$

There is an analogous formula for $I_{13}$. Note that, due to the total cancellation between the gauge field and the scalar, there is no contribution to (11) with both ends of the propagators on the same side of the polygon. Using polar coordinates we get from (12) for $p_{j} p_{k}<0$

$$
\begin{aligned}
I_{j k}^{\text {cusp }} & =2\left(b_{j k}+1\right) \int_{0}^{\pi / 4} \frac{d \varphi}{1-b_{j k} \sin 2 \varphi+i \varepsilon} \int_{\frac{\sigma_{j k}}{\cos \varphi}}^{\frac{1}{\cos \varphi}} \frac{d r}{r} \\
& =\log \sigma_{j k} \frac{b_{j k}+1}{b_{j k} \sqrt{1-1 / b_{j k}^{2}}}\left(\log \left(-b_{j k}\right)+\log \left(1+\sqrt{1-1 / b_{j k}^{2}}\right)\right)
\end{aligned}
$$

where we introduced $b_{j k}=\frac{p_{j} p_{k}}{m^{2}}$. For a later continuation to positive $p_{j} p_{k}$, one has to make more precise the issue of the phase of $b_{j k}$. We need the behaviour of $I_{j k}^{\text {cusp }}$ for 
large $b_{j k}$. The only place where this is relevant is in the term $\log \left(-b_{j k}\right)$. Due to the $i \varepsilon$ prescription in the first line of (14) this has to be understood as $\log \left(e^{i \pi} b_{j k}\right)$.

The limit for $I_{24}+I_{13}$ at $m^{2} \rightarrow 0$ is finite and can be taken from $[2,3]$. Then, expressing the products $p_{j} p_{k}$ in terms of the Mandelstam variables $\left(2 p_{1} p_{2}=2 p_{3} p_{4}=\right.$ $s+2 m^{2}, 2 p_{2} p_{3}=2 p_{1} p_{4}=t+2 m^{2}$ ), using (11), (14) and the relation of the cutoff to $m^{2}$ via (8), we arrive at

$$
W^{(1)}=-\log ^{2}\left(\frac{m^{2}}{-s}\right)-\log ^{2}\left(\frac{m^{2}}{-t}\right)+\frac{1}{2} \log ^{2} \frac{s}{t}+\frac{\pi^{2}}{2}+\mathcal{O}\left(m^{2} \log m^{2}\right) .
$$

Up to a constant independent of $s$ and $t$, this agrees with the off-shell scattering amplitude (3).

If one alternatively uses an unmodified cutoff according to (9), one gets an unwanted factor $\frac{1}{2}$ in front of the squared logarithms, as in (6) . It would be interesting to find out, whether this effect is related to the different soft regions for the scattering amplitude mentioned in [2].

\section{Comments on gauge variance/invariance}

As mentioned above, the off-shell scattering amplitude as well as our cut Wilson loop are not gauge invariant. The observed agreement of both sides concerns Feynman gauge. However, due to the role of the coefficient in front of the leading term $\left(\propto\left(\log m^{2}\right)^{2}\right)$ in the evolution equation for the scattering amplitude [2], one should expect, that the leading term in (15) is gauge invariant.

The gauge field propagator in generalized Feynman gauge with gauge parameter $\alpha$ can be written as

$$
G_{\mu \nu}^{(\alpha)}(x-y)=\frac{\eta_{\mu \nu}}{4 \pi^{2}\left((x-y)^{2}-i \varepsilon\right)}+\frac{\alpha-1}{16 \pi^{2}} \partial_{\mu} \partial_{\nu} \log \left(\Lambda^{2}(x-y)^{2}-i \varepsilon\right) .
$$

The auxiliary scale parameter $\Lambda^{2}$ drops out after performing the differentiation. Its only purpose is to start with the logarithm of a dimensionless quantity. The propagator of the scalar field is independent of $\alpha$. Therefore, the gauge dependent term to the one loop contribution $W^{(1)}$ for a generic closed contour is

$$
W_{\text {gauge }}^{(1)}=\frac{\alpha-1}{16 \pi^{2}} \int_{\tau_{1}>\tau_{2}} d \tau_{1} d \tau_{2} \frac{d}{d \tau_{1}} \frac{d}{d \tau_{2}} \log \left(\Lambda^{2}\left(x\left(\tau_{1}\right)-x\left(\tau_{2}\right)\right)^{2}-i \varepsilon\right) .
$$

Due to the $i \varepsilon$ prescription this term needs no regularization, it is identically zero before $\varepsilon$ is sent to zero. This situation changes for our loop cut according to fig.1. In addition, the limit $\varepsilon \rightarrow 0$ only exists, if before, an additional cutoff $\sigma$ is introduced, preventing the coincidence of $\tau_{1}$ and $\tau_{2}$ on the same side of the polygon. For this it would be natural to choose $\sigma=\Lambda \mathrm{m}$. Thus, to handle generalized Feynman gauge we pick up another scale parameter $\Lambda$ for dimensional reasons. But in any case, due to the total derivative structure in (17), the final result for the gauge dependent contribution can contain only log-terms and no $\log ^{2}$-terms. This proves the gauge independence of the leading $\log ^{2}$-term in our one loop result of the previous paragraph. 


\section{Conclusions}

We have given various arguments for a regularization of polygonal Wilson loops that exactly matches the MHV off-shell gluon amplitudes. This is true up to finite terms independent of the Mandelstam variables and terms vanishing for $m^{2} \rightarrow 0$. While for light-like polygons there is no difference between the pure gauge field Wilson loop and the local supersymmetric version, our off shell extension is based on the supersymmetric loop. The proposal has been checked explicitly on 1-loop level for the four gluon case. Furthermore, it has been shown, that the leading $\log ^{2}$ contribution is independent of the gauge parameter of generalized Feynman gauge.

An obvious continuation of this work should be the inclusion of higher orders in perturbation theory. Our regularization could also be of interest in the discussion of the dual conformal symmetry of scattering amplitudes and Wilson loops $[2,4,8]$.

Turning to the strong coupling situation, one should construct a regularization for the string approaching an off light cone polygon at the boundary of AdS. There an alternative to dimensional regularization, but still for the light-like case, has already been discussed in [7]. It uses a cutoff in the radial direction which is position dependent similar to our construction.

\section{Acknowledgement}

This work has been supported in part by the German Science Foundation (DFG) grant DO 447/4-1. We thank Nadav Drukker, George Jorjadze, Jan Plefka, Arkady Tseytlin and Donovan Young for useful discussions and Gregory Korchemsky for a helpful e-mail.

\section{References}

[1] L. F. Alday and J. M. Maldacena, JHEP 0706 (2007) 064 arXiv:0705.0303 [hepth]].

L. F. Alday and J. Maldacena, JHEP 0711 (2007) 068 [arXiv:0710.1060 [hep-th]].

[2] J. M. Drummond, G. P. Korchemsky and E. Sokatchev, Nucl. Phys. B 795 (2008) 385 arXiv:0707.0243 [hep-th]].

[3] A. Brandhuber, P. Heslop and G. Travaglini, Nucl. Phys. B 794 (2008) 231 arXiv:0707.1153 [hep-th]].

[4] J. M. Drummond, J. Henn, G. P. Korchemsky and E. Sokatchev, Nucl. Phys. B 795 (2008) 52 arXiv:0709.2368 [hep-th]].

[5] J. M. Maldacena, Phys. Rev. Lett. 80 (1998) 4859 arXiv:hep-th/9803002.

[6] N. Drukker, D. J. Gross and H. Ooguri, Phys. Rev. D 60 (1999) 125006 arXiv:hep-th/9904191. 
[7] L. F. Alday, "Lectures on Scattering Amplitudes via AdS/CFT," arXiv:0804.0951 [hep-th].

[8] J. M. Drummond, J. Henn, G. P. Korchemsky and E. Sokatchev, "Conformal Ward identities for Wilson loops and a test of the duality with gluon amplitudes," arXiv:0712.1223 [hep-th].

J. M. Drummond, J. Henn, G. P. Korchemsky and E. Sokatchev, "Dual superconformal symmetry of scattering amplitudes in $\mathrm{N}=4$ super-Yang-Mills theory," arXiv:0807.1095 [hep-th]. 